\title{
Quality of clinical trials for selected priority mental and neurological disorders in sub-Saharan Africa: a systematic review
}

This article was published in the following Dove Press journal:

Open Access Journal of Clinical Trials

7 December 2016

Number of times this article has been viewed

\author{
Anwar Mulugeta' \\ Girmay Medhin² \\ Getnet Yimer' \\ Rahimush Jemal ${ }^{3}$ \\ Abebaw Fekadu ${ }^{4,5}$ \\ 'Department of Pharmacology, \\ School of Medicine, College of \\ Health Sciences, Addis Ababa \\ University, ${ }^{2}$ Aklilu Lemma \\ Institute of Pathobiology, Addis \\ Ababa University, ${ }^{3}$ Department of \\ Pharmacy, Tikur Anbesa Hospital, \\ ${ }^{4}$ Department of Psychiatry, School \\ of Medicine, College of Health \\ Sciences, Addis Ababa University, \\ Addis Ababa, Ethiopia; ${ }^{5}$ Department \\ of Psychological Medicine, Centre \\ for Affective Disorders, Institute of \\ Psychiatry, King's College London, \\ London, UK
}

Background: There is a developing consensus on the effectiveness of various interventions for mental disorders in low- and middle-income countries, and it has been proposed that the main task is to scale up these interventions. In this context, we aimed to review the quality and extent of intervention trials for selected priority mental and neurological disorders in sub-Saharan Africa. Methods: Medline and African Journals Online databases were used for searching relevant articles. Both randomized and nonrandomized clinical trials for the treatment of schizophrenia, depression, maternal depression, bipolar disorder, and epilepsy/seizure disorders that involve pharmacotherapy, psychotherapy, and physical therapy were included. An extensive list of search terms that identified locations, disorders, interventions, and study types were employed. The qualities of the trials were appraised using the single-component quality assessment of the consolidated standards of reporting trials (CONSORT) statement and the Jadad scale.

Results: From 1,136 studies identified, only 34 trials that fulfilled inclusion criteria were used for quality analysis. Most studies were clinical trials of treatments for epilepsy and were conducted after 2006. In terms of region, the majority of studies were conducted in South Africa (22 of the 34 studies). Approximately half of the trials (53\%) were conducted in single center and the majority of the trials $(71 \%)$ were pharmacotherapeutics-based intervention. In terms of methodological quality in relation to the Jadad scale, $82 \%$ fulfilled the criteria for good methodological quality with a score of $3-5$. However, the methodological quality according to the CONSORT criteria was more mixed.

Conclusion: The overall quality of clinical trials conducted in sub-Saharan Africa is encouraging despite the limited number of studies. However, important quality limitations remain and have not improved over time. Hence, establishing clinical trial centers in these countries may be one approach to improving quality and quantity of trials.

Keywords: randomized controlled trial, schizophrenia, depression, epilepsy, drug therapy, psychotherapy, Africa

\section{Introduction}

For the most part, interventions that have proven efficacy in high-income countries are imported and applied in low- and middle-income countries (LAMICs). ${ }^{1}$ This may be acceptable in a general medical treatment context where standard causative pathogens or established pathological processes with an established pattern of treatment responsiveness lead to a disease condition. The main consideration in a general medical treatment context would be individual variations that may occur due to differences in genetic makeup and consequent variations in pharmacokinetic and pharmacodynamic processes of drugs. ${ }^{2}$ Such variation also has potential impact in relation to pharmacotherapeutic interventions
Correspondence: Anwar Mulugeta Department of Pharmacology, School of Medicine, College of Health, Addis Ababa University, Zambia Street, Addis Ababa, Ethiopia

Email anwar.mulugeta@aau.edu.et 
of mental disorders. For example, CYP 2D6, phase I enzyme involved in the metabolism of most antipsychotic and antidepressant drugs, is highly polymorphic and has shown significant individual and ethnic variations. ${ }^{3}$ Aklillu et al's study from Ethiopia has supported such polymorphic variations. ${ }^{4}$ The implication of this is mainly that, in clinical practice, tolerability and required dosing of medications may depend on the origin and ethnicity of the individual being treated. Clinicians need to take such variations into account. The practice of approving the use of new drug in populations where it has not yet been studied, with the perspective of "if it fits for most it fits or all", should not be relied upon. However, in addition to the above considerations, the onset and treatment responsiveness of mental disorders may be affected by sociocultural contexts and settings. These factors have substantial bearing on the effectiveness of interventions. For example, cognitive behavioral therapy is likely to require extensive adaptation in terms of the modality of delivery and content when applied to LAMIC settings. ${ }^{5}$ Group therapies in general and talking therapy, in particular, may be affected by cultural norms of openness and acceptability of group settings. Although there is a growing conviction that there is enough evidence to scale up services in low-income countries, ${ }^{1}$ it is not entirely clear whether there are sufficient data establishing efficacy and effectiveness of interventions in LAMICs, particularly in sub-Saharan Africa. It is, therefore, important to conduct clinical trials in these settings. However, conductance of clinical trials has been facing challenges in sub-Saharan African countries. ${ }^{6}$ The challenges are partly shared by the nature of clinical trials: the high cost, the need for high levels of expertise and more complex facilities, or it could be partly because of the lack of policy that entertains such studies in the region. ${ }^{6}$

Africa is the second largest continent with $13 \%$ of the world population. ${ }^{7}$ It is a region characterized by low income, high prevalence of communicable diseases, malnutrition, low life expectancy, and poorly staffed services. Yet, Africa is also a region with unparalleled opportunities for conducting controlled trials in recent years mainly because of the economic rising of the region and encouraging policy changes in relation to clinical trials. ${ }^{8}$ Until recently, mental disorders have received little attention in the region where the major reasons of mortality remain infectious diseases and malnutrition. ${ }^{9}$ However, mental disorders are among the most prevalent classes of chronic diseases in the general population. ${ }^{10}$ Neuropsychiatric disorders, globally, account for more than a quarter of all health losses due to disability, more than eight times greater than that attributed to coronary heart disease and 20 -fold greater than cancer. ${ }^{11}$
More research is required to understand the impact and best treatment approaches for mental disorders. ${ }^{12}$ At present, the contribution of LAMICs to internationally accessible mental health literature is less than $6 \% .{ }^{13,14}$ Specifically, among the total of 13,258 publications from LAMICs indexed in Medline and PsycINFO in 2007, only $16.6 \%$ of them were from Africa, with the highest share coming from South Africa $(30 \%) .{ }^{15}$ Moreover, there is criticism that research in LAMICs does not address mental health disorder as the prioritized public health concern. ${ }^{16}$ This assumption may marginalize trials for mental disorders.

Unlike nonexperimental study designs, controlled trials are financially more costly and ethically more sensitive. As a result, most of the controlled trials have been conducted in high-income countries like the US and European countries. According to Clinicaltrial.gov database (Clinicaltrials.gov), $41.6 \%$ and $24 \%$ of the registered trials were conducted in the US and Europe, respectively, while only $2 \%$ were registered from Africa ${ }^{17}$ Less than $1 \%$ of 11,501 randomized clinical trials (RCTs) on schizophrenia, depression, alcohol use disorder, and child developmental disorders conducted in the world have been carried out in LAMICs. ${ }^{18}$

RCTs are considered the gold standard for a generation of evidence. However, ensuring a standard quality of RCTs from the planning to the execution and analysis of data is essential to make sure the study procedures are safe for participants and the conclusions that result from the RCTs are accurate. ${ }^{19}$ Establishing a system for adequate quality requires extensive infrastructure and human capacity.

Defining quality is vague and subjective, but in assessing the quality of controlled trials it is necessary to give due attention to the internal and external validity of the trials. ${ }^{19}$ From the perspective of Jadad et al, quality is "the likelihood of the trial design to generate unbiased result"; however, this definition emphasizes methodological quality. ${ }^{20}$ The consolidated standards of reporting trials (CONSORT) statement is the most comprehensive procedure to evaluate the quality of a clinical trial. ${ }^{21}$ Different articles have used Jadad scale for methodological quality assessment that addressed the method of randomization, blinding of the study, use of the term "randomization" in the manuscript, and the number and reasons of dropout. ${ }^{19,20}$ The CONSORT statement has a complementary role to Jadad, in that it also addresses a holistic overview of reporting qualities including reporting quality of the trial's title, abstract, background, methodology, result, discussion, limitation, and conclusion sections and also registration on trial database. ${ }^{21}$

Evidence-based medicine is predominantly and preferentially dependent on meta-analysis and systematic reviews 
that were formulated based on the quality levels of the trial included. However, many reviews have documented deficiencies in quality reports of clinical trials. ${ }^{22}$

Considering the increasing interest in the scale up of mental healthcare in sub-Saharan African countries, this study looked at the quality of RCTs for selected or prioritized mental disorders. The priority disorders for this review (depression, schizophrenia, and epilepsy/seizure) were selected based on the World Health Organization criteria and priorities set out by countries in sub-Saharan Africa. ${ }^{23}$ Moreover, considering the need for strong research infrastructure to conduct clinical trials, we considered that RCTs would serve as indirect indicators of the overall research capacity of sub-Saharan Africa. Given the broader challenges and system-level demands of conducting clinical trials, we hypothesized that there may not be an adequate number of trials of standard quality conducted in the region. We also hypothesized that studies conducted since the release of the CONSORT statement in 1996 are likely to be of better quality than trials conducted previously.

\section{Objectives}

The primary objective of this review was to assess the extent and quality of clinical trials for selected priority mental disorders conducted in sub-Saharan African countries. The secondary objectives were: 1) to determine the association between characteristic of included studies (year of publication, residence of the author, study country, and study disorder) and the quality of controlled trials, and 2) to identify the gaps and potential ways forward in supporting quality improvement in clinical trials in the region.

\section{Methods}

\section{Literature search}

The literature for this review was identified through a search in Medline, African Journals Online, and Google Scholar from August 2013 to January 2014. In addition, the OpenGrey database (http://www.opengrey.eu/) was used for searching abstracts, conference proceedings, and other gray literature. An extensive list of medical subject heading terms in various permutations based on the selected disorders (depression [including maternal depression/postpartum depression/prenatal depression/postnatal depression], schizophrenia, bipolar disorder, and epilepsy), a list of treatments (pharmacological, psychological/psychosocial, and physical), research designs, and list of sub-Saharan regions and countries was used. Relevant references were also searched manually for articles on the subject. Considering the fact that most of the drugs for
Box I Inclusion criteria.

- Study design: controlled trials (both randomized and nonrandomized)

- Type of participant: all age groups and genders

- Disorders: depression, including maternal depression, schizophrenia, bipolar disorder, and epilepsy/seizure

- Study setting: trials in sub-Saharan Africa countries; multinational trials that include at least one sub-Saharan Africa country in the study

- Types of publications: published and unpublished research

- Language of publication: English

- Types of interventions: drug interventions, micro-nutrition or nutritional therapy intervention, and interventions like electroconvulsive therapy, deep brain stimulation or neurosurgery therapy, and psychosocial interventions were included. stimulation or neurosurgery therapy, and psychosocial interventions were included. The type of control group could be placebo controlled, active control, or both of them

mental disorders were marketed after the 1950s, we searched only for studies published since 1950. Publications were screened first by titles and then abstracts to exclude any obvious non-eligible trials. Then, the full version of eligible publications was screened using the inclusion and exclusion criteria checklist (Box 1) to finally select the publications for analysis.

\section{Study selection}

The inclusion criteria are as shown in Box 1.

\section{Data extraction and management}

From each publication, general characteristics and quality indicators of the trials were extracted using preprepared extraction forms. These general characteristics included: setting of the trial, years of publications, study disorders, type of interventions, intervention period, funding, and characteristics of principal investigators (eg, field of specialty). A checklist derived from the CONSORT statement that addresses the major quality variables such as randomization, blinding, registration on trial database, and financial support was used to assess the reporting quality of the trials. Data were extracted by one individual (AM) trained on the CONSORT statement, Jadad scale, and the remaining extraction formats.

\section{Outcomes}

The quality of the included studies was appraised in two ways: the CONSORT guidelines for reporting quality ${ }^{21}$ and the Jadad scale for methodological quality assessment. ${ }^{20}$ The CONSORT statement includes a checklist of 22 items and flow diagrams that pertain mainly to the methods, results, and discussion of an RCT report and identifies key pieces of information necessary to evaluate the internal and external 
validity of the report. ${ }^{21}$ Reporting quality of the pharmacological intervention, non-pharmacological intervention (surgery, technical procedures, device, psychotherapy, behavioral intervention, nutritional intervention, and complementary and alternative medicine), non-inferiority, equivalence, and clustered trials was therefore assessed using the CONSORT statement.

The Jadad scale assesses the quality of published controlled trial methods relevant to random assignment, double blinding, and the flow of patients. ${ }^{20}$ Jadad scale is a simple seven-item scale, with five positively rated and two negatively rated items. The overall score ranges from 0 to 5 . A score of 3 and above is generally considered to be an indicator of good quality. Further details are provided in Figure S1.

\section{Data analysis}

Once the data were extracted and checked for accuracy, the descriptive analysis was conducted using SPSS version 16
(SPSS Inc., Chicago, IL, USA). Chi-square test was used to determine the association between methodological quality and characteristics of the trials. In all analysis, $P$-value $<0.05$ was used to decide if associations or differences were statistically significant.

\section{Results \\ General characteristics of included studies}

A total of 1136 articles were obtained from the primary search in which only 58 articles were selected for evaluation of the full article. From these 58 articles, 36 articles fulfilled the inclusion criteria but only 34 articles were extracted and analyzed for their quality. The full version of two articles could not be accessed despite the effort to get it through mail communication with the authors (Figure 1). The articles published in 23 different journals have an impact factor that ranks from 0.871 to 51.658 (three articles' journal impact factor is

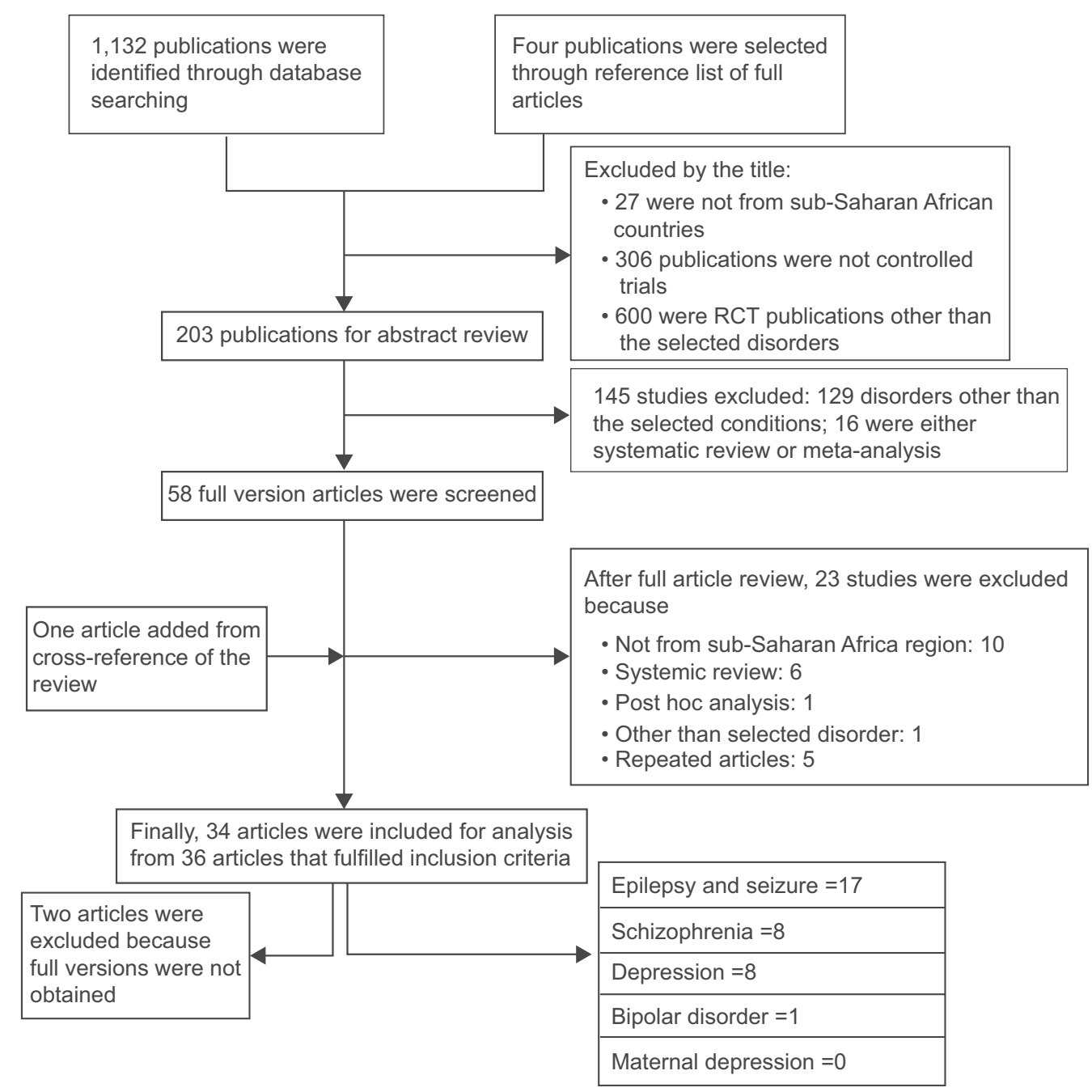

Figure I Flow diagram of selection of trials on selected priority disorders in sub-Saharan Africa. Abbreviation: RCT, randomized clinical trial. 
not included because it is not stated in the journal). Sixty-five percent of the trials were published after the year 2007, and most were from South Africa. Half of the 34 trials included people with epilepsy or infection/pregnancy-induced seizure. The majority of the trials were a pharmacological intervention $(71 \%)$ and in a single center $(53 \%)$. Fifty percent of the trials were placebo controlled and the majority (79\%) of the trials involved two groups for comparison. Regarding the principal investigators of the articles, $47 \%$ were medical doctors, $18 \%$ were nurses and public health professionals, while the remaining $35 \%$ trials did not state the authors' profession clearly. Out of the 34 included trials, $30 \%$ of them were reported by authors based in Africa, $32 \%$ only by authors based outside Africa, and $38 \%$ by authors based in Africa and elsewhere (data not shown). The initial sample size recruited for the interventions range from a minimum of 20 to a maximum of 629 participants, while the median dropout number of participants was 24. Only 6 of 34 trials had 50 and fewer participants (Table 1).

The intervention period for the trials was diverse, comprising a single-dose administration of the study drug with 56 weeks of intervention period and with the mean \pm standard deviation of $24.4 \pm 4.5$ weeks. In this analysis, trials with single-dose administration of drugs (four trials) and missed values (three trials) were not considered (Figure 2).

Diverse age groups were observed in the studies. There were clinical trials conducted in infants (mean age of 2.5 years) through to elderly population (mean age of 71.1 years). The pooled average age of the participant in the included study was 32.1 years (Table 1).

\section{Report quality of clinical trials based on the CONSORT statement}

Only one article met the full reporting quality criteria in all components of the CONSORT statement. The lowest rate of reporting quality (from the articles) was $36 \%$ (Figure 3 ). The highest reporting concordance $(100 \%)$ was observed in the reporting of primary and/or secondary outcomes and statistical methods for primary and/or secondary outcomes. The lowest report compliance was observed in the registration of clinical trials on databases, in which only 8 of the 34 articles were registered. Regarding reporting on methodological components of the CONSORT statement, the study design was described in $71 \%$, double blinding in $65 \%$, and adequate method of blinding in $47 \%$ of articles. From 24 articles which reported on sequence generation, the most common methods of sequence generation were computer-generated randomization number list (17 of 24). However, only 11 of
Table I Characteristics of the trials on selected mental and neurological disorders in sub-Saharan African countries

\begin{tabular}{|c|c|c|}
\hline $\begin{array}{l}\text { Characteristics of trials } \\
\text { (categorical variable) }\end{array}$ & Descriptions & n (\%) \\
\hline \multirow[t]{3}{*}{ Year of publication } & $\leq 1996^{a}$ & $2(6)$ \\
\hline & $(1996-2006)$ & $10(29)$ \\
\hline & $>2006^{\mathrm{b}}$ & $22(65)$ \\
\hline \multirow[t]{4}{*}{ Study country region } & Southern Africa & $22(64)$ \\
\hline & East Africa & $9(26)$ \\
\hline & West Africa & $2(6)$ \\
\hline & Central Africa & I (3) \\
\hline \multirow[t]{5}{*}{ Study disorder } & Epilepsy/seizure & $17(50)$ \\
\hline & Schizophrenia & $8(23.5)$ \\
\hline & Depression & $8(23.5)$ \\
\hline & Bipolar disorder & $\mathrm{I}(3)$ \\
\hline & Maternal depression & 0 \\
\hline \multirow[t]{4}{*}{ Intervention type } & Pharmacological & $24(7 I)$ \\
\hline & Non-pharmacological & $10(29)$ \\
\hline & Psychotherapy & $9(26)$ \\
\hline & $\begin{array}{l}\text { ECT and brain } \\
\text { stimulation }\end{array}$ & I (3) \\
\hline Type of intervention with & Placebo-controlled & $17(50)$ \\
\hline respect to the control & Active-controlled & $13(38)$ \\
\hline group & Both & $4(12)$ \\
\hline Number of study groups & Two groups & $27(79)$ \\
\hline (intervention and control & Three groups & $5(15)$ \\
\hline \multirow[t]{2}{*}{ group) } & Four groups & I (3) \\
\hline & Five groups & I (3) \\
\hline Number of data collection & Single center & $18(53)$ \\
\hline \multirow[t]{2}{*}{ sites } & Multicenter/multinational & $15(44)$ \\
\hline & Not clear & I (3) \\
\hline Treatment/intervention & Yes & $30(88)$ \\
\hline effective & No & $4(12)$ \\
\hline
\end{tabular}

Characteristic of

principal investigator

Affiliated institutions

University $22(64)$

Research center $3(9)$

Pharmaceutical company $2(6)$

Health institutions $\quad 7(2 \mathrm{I})$

Field of specialty $\quad$ Medical doctor $\quad 16(47)$

Other health 5 (15)

professionals

Not specified

II (32)

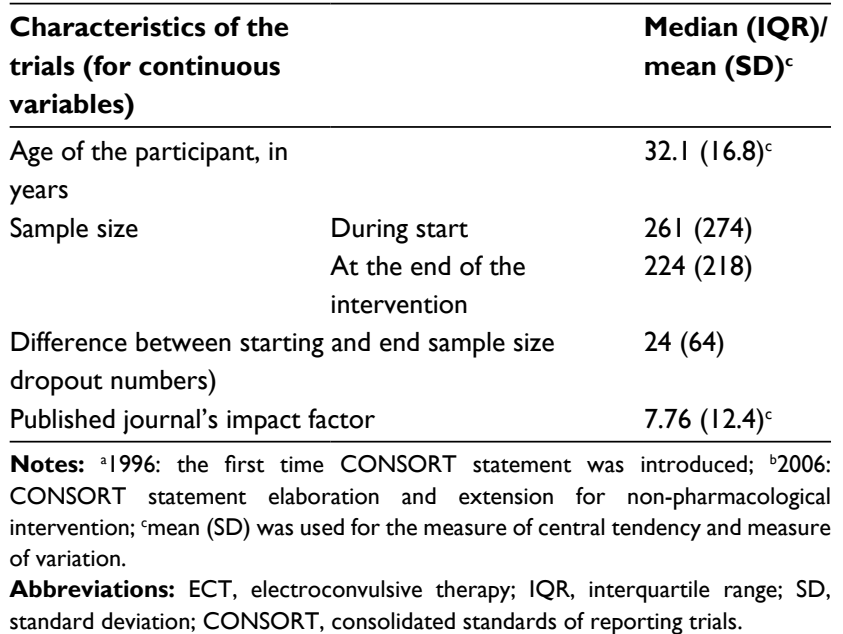

Abbreviations: $E C T$, electroconvulsive therapy, $\mathrm{QR}$, interquartile range; $S D$, standard deviation; CONSORT, consolidated standards of reporting trials. 


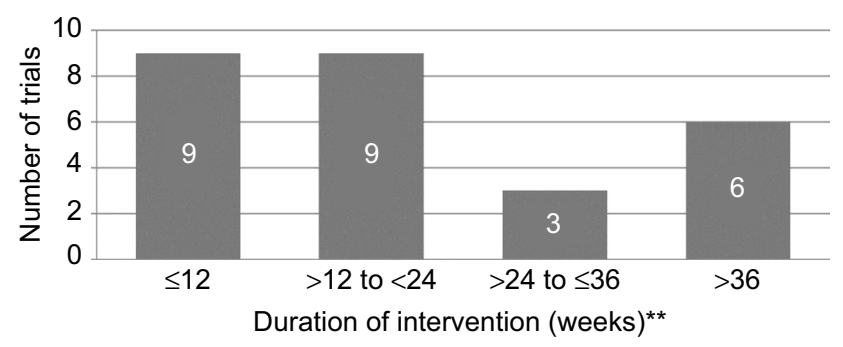

Figure 2 Frequency distribution of clinical trials based on the intervention period. Note: **The duration of intervention in weeks included in the graph does not include trials with single-dose administration (four trials) and trials in which the period is not stated (three trials).

the 34 articles reported allocation concealment, in which the common methods of allocation concealment were interactive voice recording system (5 of 11) and sealed envelope (4 of 11). In $38 \%$ and $68 \%$ of the trials, the method of randomization and method of allocation concealment, respectively, were not mentioned (Table 2).

\section{Quality of controlled trial methodology using Jadad score}

As shown in Figure 4, most of the articles (82\%) had Jadad score of 3 or more, suggesting the majority of the studies were of the acceptable quality standard.

\section{Discussion}

To our knowledge, this is the first evaluation of the quality of RCTs in Africa. The main findings of our review were: 1) the number of clinical trials conducted in Africa in the field of mental health are very few; 2) of these, most clinical trials were conducted in South Africa; 3) the methodological quality of the trials, according to the Jadad scale, was encouraging with over three-quarters having acceptable methodological quality; 4) similarly, the reporting quality according to the CONSORT statement was also encouraging; and 5) over time, the reporting quality of clinical trials has not improved and this is of some concern. The scope of trial types and treatment options was also limited. For example, the initial expectation of the search was also to include intervention types that included pharmacological psychotherapy (psychotherapy including behavior, cognitive, and cognitive behavioral therapies), nutritional therapy, and other therapies (electroconvulsive therapy, deep brain stimulation, and surgery); however, only pharmacological interventions, psychotherapy, and electroconvulsive therapy trials were found.

The findings of this review reinforce the view that lowincome countries, specifically sub-Saharan African countries are underrepresented in clinical research. The disparity in research and service capacity was assessed using an index called clinical trials index (CTI), which is computed by simply estimating "the share of clinical trials a country or a region has registered in clinical trial registry databases". Though Africa comprises $13 \%$ of the world population, it has contributed to only $2 \%$ of the clinical trials in the world, whereas the US took $41 \%$ of the share. Of the few studies conducted in Africa, more than half of these were done in Egypt and South Africa (CTI $=64.1 \%$ ). The contribution of majority of African countries, especially sub-Saharan African countries, to the total pool of world clinical trials is insignificant. ${ }^{24} \mathrm{~A}$ broad set of challenges contribute to the scarcity of clinical research in Africa. Although these challenges partly relate to the unique requirements of conducting clinical trials, these broader challenges are relevant to clinical research in general. These challenges include lack of appropriate infrastructure, for example, space, supplies, and maintenance; weak administrative capacity;

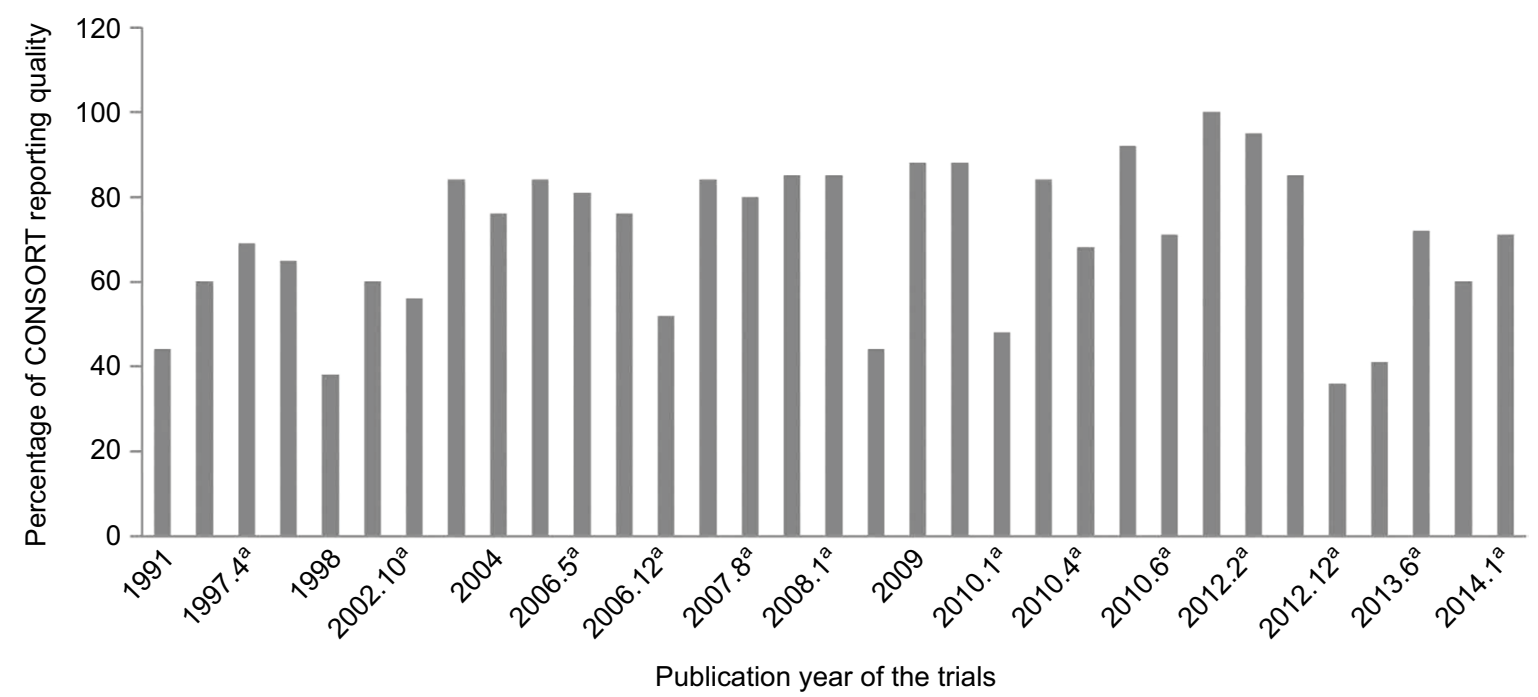

Figure 3 Description of the trials' CONSORT reporting quality over time (I99| to 2014).

Note: anenotes publication year of the trials followed by the specific month (2008.I is a publication in January 2008).

Abbreviation: CONSORT, consolidated standards of reporting trials. 
Table 2 Reporting quality of the controlled trial on selected mental and neurological disorders in sub-Saharan African countries

\begin{tabular}{|c|c|c|}
\hline \multicolumn{2}{|c|}{ Characteristics } & Yes, n (\%) \\
\hline \multicolumn{2}{|c|}{ Identification as a randomized trial in the title } & $21(62)$ \\
\hline \multicolumn{2}{|c|}{ Specific objectives or hypothesis were specified } & $33(97)$ \\
\hline \multicolumn{2}{|c|}{ Description of eligibility criteria for participants } & $33(97)$ \\
\hline \multicolumn{2}{|c|}{ Participant flow diagram } & $17(50)$ \\
\hline \multicolumn{2}{|c|}{$\begin{array}{l}\text { Description of settings and locations where the data } \\
\text { were collected }\end{array}$} & $30(88)$ \\
\hline \multicolumn{2}{|c|}{$\begin{array}{l}\text { Adequate description of design (eg, placebo-controlled, } \\
\text { parallel, factorial, superiority, and non-inferiority) }\end{array}$} & $24(7 I)$ \\
\hline \multicolumn{2}{|c|}{ Clear sample size calculation } & $20(59)$ \\
\hline \multirow[t]{2}{*}{ Outcomes } & $\begin{array}{l}\text { Primary/secondary outcomes } \\
\text { mentioned }\end{array}$ & $34(100)$ \\
\hline & $\begin{array}{l}\text { Specify when and how the } \\
\text { outcomes assessed }\end{array}$ & $32(94)$ \\
\hline \multirow[t]{2}{*}{ Intervention } & $\begin{array}{l}\text { Specify how long the intervention } \\
\text { stayed }\end{array}$ & $33(97)$ \\
\hline & $\begin{array}{l}\text { Specify how and at what dose the } \\
\text { intervention was done }\end{array}$ & $24(7 I)$ \\
\hline \multirow[t]{2}{*}{ Randomization } & Sequence generation & $21(62)$ \\
\hline & Allocation concealment & II (32) \\
\hline \multicolumn{2}{|c|}{ Double blinding } & $22(65)$ \\
\hline \multicolumn{2}{|c|}{ Adequate method of blinding } & $16(47)$ \\
\hline \multicolumn{2}{|c|}{ Intent to treat } & $22(65)$ \\
\hline \multicolumn{2}{|c|}{$\begin{array}{l}\text { Statistical methods for primary and/or secondary } \\
\text { outcome used }\end{array}$} & $34(100)$ \\
\hline \multicolumn{2}{|c|}{$\begin{array}{l}\text { Method of additional analysis used like subgroup } \\
\text { analysis or adjusted analysis }\end{array}$} & $21(62)$ \\
\hline \multicolumn{2}{|c|}{ Define date (period) of recruitment } & $17(50)$ \\
\hline \multicolumn{2}{|c|}{$\begin{array}{l}\text { Present a table that shows demographic and clinical } \\
\text { characteristics }\end{array}$} & $30(88)$ \\
\hline \multicolumn{2}{|c|}{ Estimated effects and their precision presented } & $26(77)$ \\
\hline \multicolumn{2}{|c|}{ Harms or unintended effects presented/specified } & $26(77)$ \\
\hline \multicolumn{2}{|c|}{ Limitations specified } & $20(59)$ \\
\hline \multicolumn{2}{|c|}{ Registration } & $8(24)$ \\
\hline \multicolumn{2}{|c|}{ Has funding source } & $21(62)$ \\
\hline
\end{tabular}

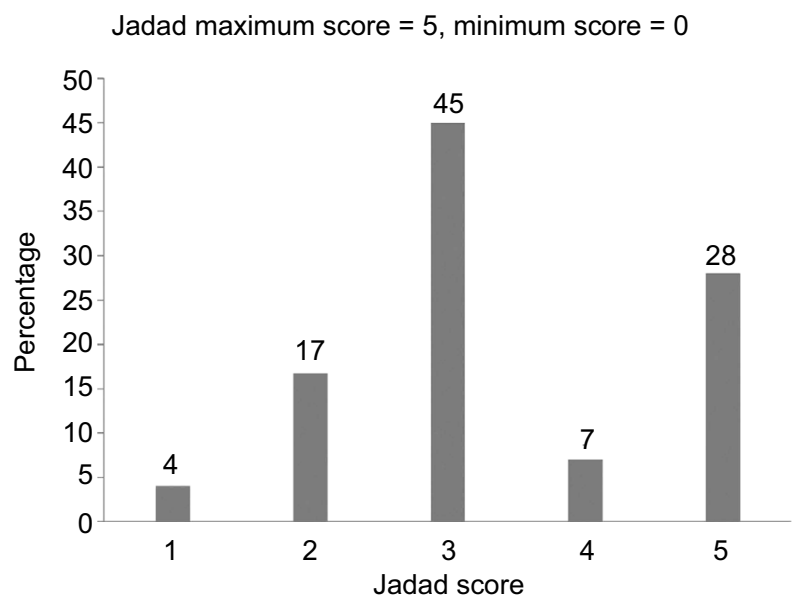

Figure 4 Quality of clinical trial using validated Jadad score, scores of 3 and above indicate acceptable quality standard. lower prioritization of research in academic institutions; limited university-industry linkage; limited sources of funding; and the lack of incentives for researchers. ${ }^{6,25}$ Clinical trials also require unique skills among trial teams, and developing these skills takes a very long time. Moreover, once this capacity is developed, brain drain is another frequent threat. ${ }^{26}$ However, investing in clinical trials is essential for Africa, given the great potential for clinical trials in the continent, diversity of the population, and the need to have locally contextualized evidence. ${ }^{2}$ Such locally contextualized evidence is particularly important for psychosocial interventions, which are affected by local context more than any other disease. ${ }^{5}$ Randomized controlled trials are the best approaches to developing robust and locally relevant evidence. ${ }^{27} \mathrm{~A}$ more pressing gap in Africa is the extremely few numbers of professionals in the discipline within the region. ${ }^{28}$

Most of the trials (22 of 34) were conducted in Southern Africa, all conducted in South Africa, and followed by East Africa (9 of 34). The high number of studies from South Africa is concordant with the CTI for South Africa and is the result of the relatively large financial investments on health the country makes. ${ }^{25}$ Even for clinical trials conducted in South Africa, most were for epilepsy and seizure (13 of 22), a primarily neurological condition. Similarly, a third of the studies conducted in East Africa (3 of 9) were for epilepsy. Moreover, the influence of other players in these clinical trials can also be observed. In South Africa, nearly half of the studies conducted were part of multicenter and multinational trials, most of which involved drug company-sponsored trials.

Despite the small number of trials identified, our study found that the quality of the methodology and reporting was overall encouraging. In terms of the methodological quality, over three-quarters of the included studies ( $82 \%)$ had received good methodological quality according to the Jadad score. However, the methodological quality of the studies showed little improvement over time, with five of the six studies of poor quality having been conducted after 2006. However, Jadad scale does not include randomization concealment as a quality criterion. This is an important limitation. For example, in this report, 14 trials of 23, which have good quality on the Jadad scale, did not mention the method of concealment of the randomization process. Therefore, the high Jadad scale score might be a little misleading.

In terms of reporting quality, the review indicates that a good proportion of studies have used the reporting criteria of the CONSORT (the single-component quality assessment). The areas where the reporting quality was particularly good was on reporting of primary/secondary outcomes, statistical 
methods for primary and/or secondary outcomes, participants' eligibility criteria, setting of objective/hypothesis, duration of the intervention, and when and how the outcomes were assessed. Nevertheless, there were important shortcomings in many of the studies. For example, almost two-thirds of the studies did not mention "randomization" in the title, while none of the cluster trials $(n=8)$ mentioned "cluster" in the title. Ensuring internal and external validity of trials is a key for reducing the risk of bias, and the main strategies for improving validity are double blinding, sequence generation, and randomization concealment. ${ }^{19}$ The proportion of studies that have adhered to standard sequence generating procedures $(62 \%)$ was much higher than what has been reported of PubMed-indexed trials in 2000, where only $21 \%$ followed standard procedure, and those indexed in 2006, where only $34 \%$ followed standard sequencing procedure. ${ }^{29}$ However, allocation concealment was reported in only 11 of 34 articles. Although the lack of reporting does not necessarily mean that allocation was not concealed in all cases, it is indicative of an important problem that reduces the value of the sequence generation. ${ }^{30}$ It is of note that trials with inadequate generation of allocation sequence and/or inadequate method of allocation concealment yielded inflated treatment effects. ${ }^{31,32}$

Adequate sample size and statistically valid sample size calculation are vital for getting reliable intervention effect and assuring the external validity of the trial. Although some studies have used as few as 20 participants in their trials, the median (interquartile range) number of participants was 260 (69-343). This compares favorably to international studies in schizophrenia where $50 \%$ of clinical trial studies had 50 or fewer participants. ${ }^{33}$ This is indicative of the opportunity for conducting better-powered studies in Africa.

\section{Limitations}

The CONSORT statement checklist is specifically designed to provide guidance to authors to improve reporting quality of trials, but it does not address facets of reports that require attention, such as scientific content and reliability of RCT reports. ${ }^{34}$ On the other hand, the lack of allocation concealment domain in the Jadad scale is the main limitation of the Jadad scale tool ${ }^{19}$; however, the use of CONSORT statement along with Jadad scale will result in a more comprehensive and complimentary assessment of methodological and reporting quality of the trials. Extraction and screening were carried out by the single reviewer, which is the limitation of the study; however, selection of articles relied on continuous discussion with two of the senior authors of this article. Once the screening and data extraction were done, it was verified by the senior researcher of this author. The inclusion of studies that were published only in the English language is another limitation of the study.

\section{Conclusion}

The extremely few number of clinical trials conducted in Africa is a major concern and indicative of the overall inequity in conducting clinical research. Improving capacity in the area of clinical research and clinical trials is an essential step for improving the health of the population. Africa has great potential for supporting clinical trials because of the large population, diversity of the population, and improving economic outlook. Contextualized evidence is particularly important for interventions of mental disorders and noncommunicable diseases. The quality of the studies conducted in Africa is also encouraging, although the lack of improvement over time may be of some concern. The quality requirements may have to be part of the evaluation framework for ethics approval, and not just requirements during publication.

\section{Acknowledgments}

The authors sincerely thank all staff members of Folate and Minos trial and Prime project for their continuous assistance in providing valuable information related to this work. Especially, they give credit to Silenat Biresaw and Melkam Alemayehu since they had been involved in searching articles and clearing data. To conduct this review, the authors did not get any support from funding agents.

\section{Author contributions}

AMG and AF conceived the study and later the idea was discussed with GM and GY for consolidating the hypothesis and then to draft the manuscript. AMG, AF, RJ, and GM were involved in collecting articles and extracting data. All the authors were involved in the design of the study, performed statistical analysis, and wrote and critically revised the manuscript. All the authors read and approved the final manuscript.

\section{Disclosure}

The authors report no conflicts of interest in this work.

\section{References}

1. Patel V, Thornicroft G. Packages of care for mental, neurological, and substance use disorders in low- and middle-income countries. PLoS Med. 2009;6:e1000160.

2. Murray M. Role of CYP pharmacogenetics and drug-drug interactions in the efficacy and safety of atypical and other antipsychotic agents. J Pharm Pharmacol. 2006;58:871-885.

3. Bertilsson L, Dahl ML, Dalen P, Al-shurbaji A. Molecular genetics of CYP2D6: Clinical relevance with focus on psychotropic drugs. $\mathrm{Br} J$ Clin Pharmacol. 2002;53:111-122. 
4. Aklillu E, Persson I, Bertilsson L, Johansson I, Rodrigues F, Ingelman-Sundberg M. Frequent distribution of ultrarapid metabolizers of debrisoquine in an Ethiopian population carrying duplicated and multiduplicated functional CYP2D6 alleles. $J$ Pharmacol Exp Ther. 1996;278:441-446.

5. Pamela A. Multicultural application of cognitive-behavior therapy. Prof Psychol: Res Prac. 1995;26:309-331.

6. Cardoso A, Breugelmans G, Manville C, et al. Africa mapping: current state of health research on poverty-related and neglected infectious diseases in sub-Saharan Africa. The Hague, Netherlands: European \& Developing Countries Clinical Trials Partnership; 2014:1-38. Available from: http://www.rand.org/pubs/external_publications/EP50529.html. Accessed October 1, 2016.

7. USAID: Population Reference Bureau. World population data sheet. 2014. Available from: http://www.prb.org/Publications/ Datasheets/2014/2014-world-population-data-sheet/data-sheet.aspx. Accessed September 13, 2014.

8. Puppalwar G, Mourya M, Kadhe G, Mane A. Conducting clinical trials in emerging markets of sub-Saharan Africa: review of guidelines and resources for foreign sponsors. Open Access $J$ Clin Trials. 2015;7:23-34.

9. African Development Indicators 1998/99. Washington, DC: World Bank; 1998. Available from: http://documents.worldbank.org/curated/ en/1998/10/727945/african-development-indicators-199899. Accessed November 2, 2014.

10. Okasha A. Mental health in Africa: the role of the WPA. World Psychiatry. 2002;1(1): 32-35.

11. Murray C, Lopez A. The global burden of disease: a comprehensive assessment of mortality and disability from diseases, injuries, and risk factors in 1990 and projected to 2020. Cambridge, MA: Harvard School of public Health on behalf of the World Health Organization and the World Bank; 1996. Available from: http://www.who.int/mip/2003/ other_documents/en/globalburdenofdisease.pdf. Accessed August 5, 2012.

12. Uznanski A, Roos J. The situation of mental health services of the World Health Organization, African Region, in the early 1990s. S Afr Med J. 1997;87:1743-1749.

13. Saxena S, Paraje G, Sharan P, Kram G, Sadana R. The 10/90 divide in mental health research: trends over a 10-year period. Br J Psychiatry. 2006;188:81-82.

14. Patel V, Sumathipala A. International representation in psychiatric literature: survey of six leading journals. Br J Psychiatry. 2001;178:406-409.

15. Sharan P, Levav I, Olifson S, de Francisco A, Saxena S, editors. Research Capacity for Mental Health in Low- and Middle-Income Countries: Results of a Mapping Project. Geneva: World Health Organization \& Global Forum for Health Research; 2007.

16. Flisher J, Parry D, Stein J. To what extent does South African mental health and substance abuse research address priority issues? SAfr Med J. 2000;90:378-380.
17. Clinicaltrial.gov. A service of the U.S. National Institutes of Health. Available from: http://clinicaltrials.gov/ct2/search/browse?brwse=cond_ cat_BXM. Accessed October 7, 2012.

18. Patel V, Araya R, Chatterjee S, et al. Global mental health 3: treatment and prevention of mental disorders in low-income and middle-income countries. Lancet. 2007;370:991-1005.

19. Jüni P, Altman DG, Egger M. Assessing the quality of controlled clinical trials: systematic reviews in health care. BMJ. 2001;323:42-46.

20. Jadad AR, Moore RA, Carroll D, et al. Assessing the quality of report of randomized clinical trial is blinding necessary? Control Clin Trials. 1996; 17:1-12.

21. Moher D, Hopewell S, Schulz KF, et al. CONSORT 2010 explanation and elaboration: updated guidelines for reporting parallel group randomised trials. BMJ. 2010;340:c869.

22. Sjögren $P$, Halling A. Quality of reporting randomised clinical trials in dental and medical research. Br Dent J. 2002;192(2):100-103.

23. Lund C, Tomlinson M, Fekadu A, et al. PRIME: a programme to reduce the treatment gap for mental disorders in five low- and middle-income countries. PLoS Med. 2012;9:e1001359.

24. ClinicalTrial.gov. Map of all studies on ClinicalTrial.gov. Available from: https://clinicaltrials.gov/ct2/search/map. Accessed October 14, 2016.

25. Fikadu A, Teferra S, Hailu A, et al. International clinical trial day and clinical trials in Ethiopia and Africa. Trials. 2014;15:493.

26. Tebeje A. Brain drain and capacity building in Africa. International Development Research Center. Available from: https://www.idrc.ca/en/ article/brain-drain-and-capacity-building-africa. Accessed October 10, 2016.

27. Ah-See KW, Molony NC. A qualitative assessment of randomized controlled trials in otolaryngology. J Laryngol Otol. 1998;112:460-463.

28. German GA. Aspects of clinical psychiatry in sub-Saharan Africa. Brit J Psychiatry. 1972;121:461-479.

29. Hopewell S, Dutton S, Yu LM, Chan AW, Altman DG. The quality of reports of randomised trials in 2000 and 2006: comparative study of articles indexed in PubMed. BMJ. 2010;340:c723.

30. Schulz KF. Randomised trials, human nature, and reporting guidelines. Lancet. 1996;348:59-68.

31. Schulz KF, Chalmers I, Hayes RJ, Altman DG. Empirical evidence of bias. Dimensions of methodological quality associated with estimates of treatment effects in controlled trials. JAMA. 1995;273: 408-412.

32. Moher D, Jones A, Cook DJ, et al. Does quality of reports of randomised trials affect estimates of intervention efficacy reported in metaanalyses? Lancet. 1998;352:609-613.

33. Thornley B, Adams C. Content and quality of 2000 controlled trials in schizophrenia over 50 years. BMJ. 1998;317:1181-1184.

34. Moher D, Schulz KF, Altman DG; CONSORT. The CONSORT statement: revised recommendations for improving the quality of reports of parallel-group randomized trials. BMC Medl Res Methodol. 2001;1:2. 


\section{Supplementary material}

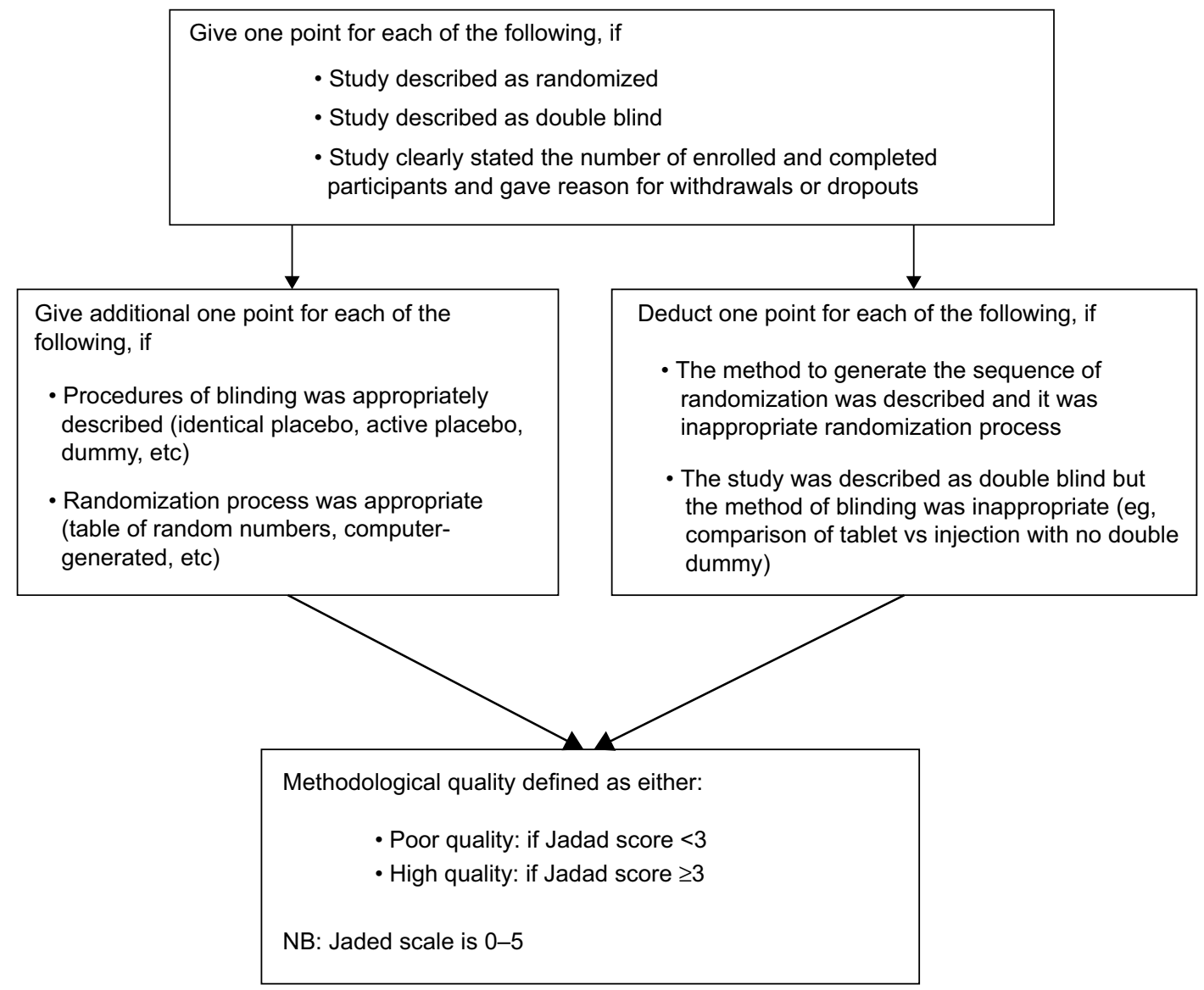

Figure SI Methodological quality assessment using Jadad scale.

\section{Publish your work in this journal}

The Open Access Journal of Clinical Trials is an international, peerreviewed, open access journal publishing original research, reports, editorials, reviews and commentaries on all aspects of clinical trial design, management, legal, ethical and regulatory issues, case record form design, data collection, quality assurance and data auditing methodologies. The manuscript management system is completely online and includes a very quick and fair peer-review system, which is all easy to use. Visit http://www.dovepress.com/testimonials.php to read real quotes from published authors. 